\title{
Insight into the Tunisian Dental Residents' Mental Wellness and Coping Mechanism during Covid-19 Pandemic: A Cross-Sectional Study
}

\author{
D. Touil, N. Aouni, F. Hannachi, F. Khanfi, L. Oualha, and N. Douki
}

\section{ABSTRACT}

Introduction: Recently, the covid-19 pandemic has dramatically affected dental students and residents worldwide. The aim of this study was to focus on the impact of the pandemic on the perceived stress, the mental health, and the coping mechanisms of the residents of the dental faculty of Monastir. Tunisia.

Materials and methods: a voluntary and anonymous survey targeting the dental residents of the Dental faculty of Monastir Tunisia, was conducted between June the $1^{\text {st }}$ to June the 30t2021. The questionnaire was divided into five sections: demographics, perceived stress during the pandemic, mental health, and coping mechanism. Yes /no questions as well as Likert scales were used.

Results: Out of the 107 residents that were contacted, 52 responded. Sex ratio was 0.48 . Among the respondents, $45.1 \%$ found that during the last 6 months, their days were "extremely stressful. A correlation between gender and daily stress was found $(p=0.046)$; concerns about the family wellbeing as well as own health and safety were ranked first when it came to evaluating the stressors during that period. Twenty-three residents $(45 \%)$ said they have had mental health problems during the last 6 months, 78\% (40) of the participants felt sad, upset, and depressed for days during the last 6 months and $31(62 \%)$ residents think they had become more irritable and in a bad mood these past few months. Talking to family and friends was the most reported coping mechanism.

Conclusion: This study has several limitations, especially the small sample size and the low return rate. However, it could be the base ground and the starting point to future studies tackling the issue of stress among Tunisian dental residents.

Keywords: Coping Mechanism, Covid-19, Pandemic, Residency, Stress, Wellbeing.
Published Online: November 19, 2021

ISSN: $2684-4443$

DOI: $10.24018 /$ ejdent.2021.2.6.113

\section{Touil*}

Dental Faculty of Monastir Research Laboratory LR12ES11, University of Monastir, Tunisia.

(e-mail: touil.oueslati.dorsaf ${ }^{\circledR}$ gmail.com) N. Aouni

Dental Faculty of Monastir Research Laboratory LR12ES11, University of Monastir, Tunisia.

F. Hannachi

Dental Faculty of Monastir Research

Laboratory LR12ES11, University of Monastir, Tunisia.

F. Khanfi

Dental Faculty of Monastir Research Laboratory LR12ES11, University of Monastir, Tunisia.

L. Oualha

Dental Faculty of Monastir Research Laboratory LR12ES11, University of Monastir, Tunisia.

N. Douki

Dental Faculty of Monastir Research Laboratory LR12ES11, University of Monastir, Tunisia.

*Corresponding Author

\section{INTRODUCTION}

Residency is an overwhelming period during which residents have tremendous responsibilities and tasks in the workplace along with their own training.

Dental residents, interns, and postgraduate students are exposed to both academic and professional stressors since they are often involved in both direct clinical care as well as scientific research. This makes them a very active component of a dental school or hospital setting. However, while being a sensitive group they still receive little attention [1]. It is common for dental residents to experience a wide range of thoughts, feelings and reactions including anxiety and fear, feeling helpless, stressed and overwhelmed [2].

Although, to some point, stress is to be expected in clinical practice and is a potentially beneficial learning stimulus, it is factual that an ideal educational environment should not be overly pressing [1]. Some reported contributing factors to resident mental wellness include lack of autonomy, environmental and workplace stressors as well as familial duties [3].

Recently, the Covid-19 pandemic has added new burden. Tunisia have been severely impacted by the pandemic with more than 25000 deaths recorded [4].

As the pandemic and its far-reaching implications continue to unfold globally, dental students and residents have been dramatically affected as they require hands-on interaction with patients which originally takes place in hospitals as well as in dental clinics. The restriction of the dental care provided, which were limited to only emergencies, resulted in the huge decrease in surgical and clinical practice and has affected residents' training. This added a new kind of stressors as residents felt uncertain about their training and the possible modification of their residency schedules [5].

To the best of our knowledge, very few studies have been performed with regards to the impact of the Covid 19 
pandemic on the Tunisian physicians' wellbeing and none on the Tunisian dental residents'.

For all these reasons, we believe that evaluating and understanding the impact of the ongoing pandemic on the Tunisian dental residents' wellbeing by focusing on its impact on their perceived mental health and their coping mechanisms while facing the pandemic's repercussions on their daily lives was a priority and motivated this study.

\section{SubJeCTS AND MethodS}

This study was based on a voluntary and anonymous survey conducted between $1^{\text {st }}$ and 30 June 2021 . The survey was sent via email to all the residents enrolled at the dental faculty of Monastir Tunisia based on name lists available at the faculty web site. Inspired by a Canadian study led by Jordan S. Cohen et al. [4], the questionnaire was adapted to fit our objectives as well as the Tunisian residency programs characteristics and then was used in this study.

The questionnaire was divided into five sections: demographics, perceived stress during the pandemic, mental health, and coping mechanism. Survey questions included qualitative rating scales, multiple choice responses as well as yes/no questions. the survey included a mixture of positively and negatively stated items in order to minimize response acquiescence bias in rating scales responses. The data was analyzed using excel Quantitative analysis including the use of student t-test and ANOVA, with statistical significance set at $\mathrm{p}<0.05$.

\section{RESULTS}

\section{A. Demographics}

Out of the 107 residents that were contacted, 52 responded giving a response rate of $48.6 \%$. The average age of the respondents was 28 years old. Twelve residents gave random birth dates. Sex ratio was 0.48 .

A total of $75.4 \%$ of the respondents $(n=40)$ were single, $15 \%(n=8)$ were married and $1.8 \%(n=1)$ were divorced (Fig2). All of the residents who responded were graduated from the Dental Faculty of Monastir. Tunisia

Table 1 reports the results of the first section of the questionnaire titled: 'Demographics'.

\section{B. Perceived Stressors during the Covid-19 Pandemic}

Forty-nine percent of the participants think that their days were "quite a bit stressful", while $45.1 \%$ answered with "extremely stressful" and $3.9 \%$ answered with "not very stressful".

A correlation between gender and daily stress $(\mathrm{p}=0.046)$ was found. No correlation between the residency program $(\mathrm{p}=0.58)$, the residency year $(\mathrm{p}=0.08)$ and stress were found.

The residents were asked to evaluate their ability to handle unexpected and difficult problems during the last few weeks, $34(66.7 \%)$ said it was poor, $14(27.5 \%)$ answered with fair and $3(5.9 \%)$ think their ability was good. None answered with excellent.

Based on the resident's responses, concerns about the family wellbeing as well as own health and safety were ranked first when it came to evaluating the stressors during that period.

Responses of the residents with regards to possible perceived stressors during the Covid-19 pandemic were summarized in the Fig. 1.

\begin{tabular}{lc}
\multicolumn{2}{c}{ TABLE I: DEMOGRAPHICS } \\
\hline \multicolumn{2}{c}{$\mathrm{N} \%$} \\
\hline Total respondents & $5248.6 \%$ \\
Gender & \\
Male & $1732.7 \%$ \\
Female & $3567.3 \%$ \\
Marital Status & \\
Single & $4075.4 \%$ \\
Married & $815 \%$ \\
Divorced & $11.8 \%$ \\
Other & $35.6 \%$ \\
Residency program & \\
General anatomy & $23.8 \%$ \\
Total Prosthodontics & $11.9 \%$ \\
Removable Partial Prosthodontics & $47.7 \%$ \\
Preventive Restorative and & $47.7 \%$ \\
Pediatric dentistry & $59.6 \%$ \\
Fixed Prosthodontics & $713.46 \%$ \\
Periodontology & $815.38 \%$ \\
Endodontics & $815.38 \%$ \\
Orthodontics & $1223 \%$ \\
Pathology and oral surgery & \\
Residency year & $1019.2 \%$ \\
$1^{\text {st }}$ year & \\
$2^{\text {nd }}$ year & \\
$3^{\text {rd }}$ year & \\
$4^{\text {th }}$ year & $1427 \%$ \\
25 h/w to 30 h/w & $2650 \%$ \\
30 h/w to 35 h/w & $1323 \%$ \\
More than 35 h/w & \\
\hline
\end{tabular}

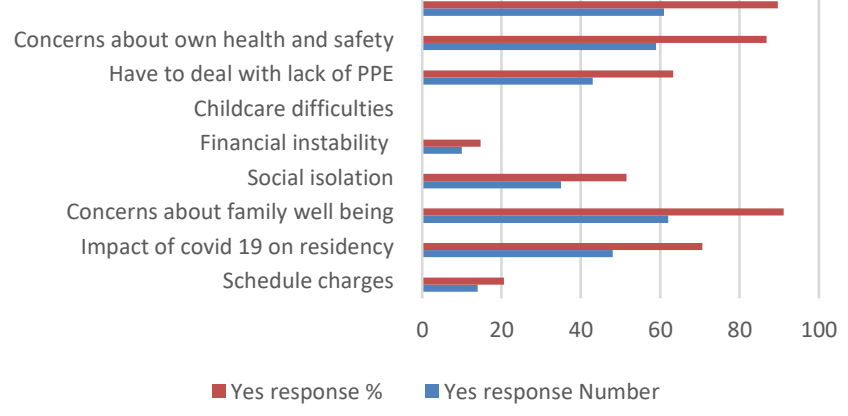

Fig. 1. Sources of stress during the Covid-19 pandemic according to the respondents.

Likert scale was used to determine the ways the residents' coping mechanism during the past 6 months.

The respondents' ways to deal with stress were reported in Fig. 2.

\section{Mental Health}

Likert scale was used to determine the residents' perceived mental health. A total of 19 respondents thought that their mental health was good and 18 thought it as fair.

Yes/no questions were asked about mental health problems. Twenty-three residents (45\%) said they have had mental health problems during the last 6 months, $78 \%$ (40) of the participants felt sad, upset, and depressed for days during the last 6 months and 31 (62\%) residents think they had become more irritable and in a bad mood these past few months. 


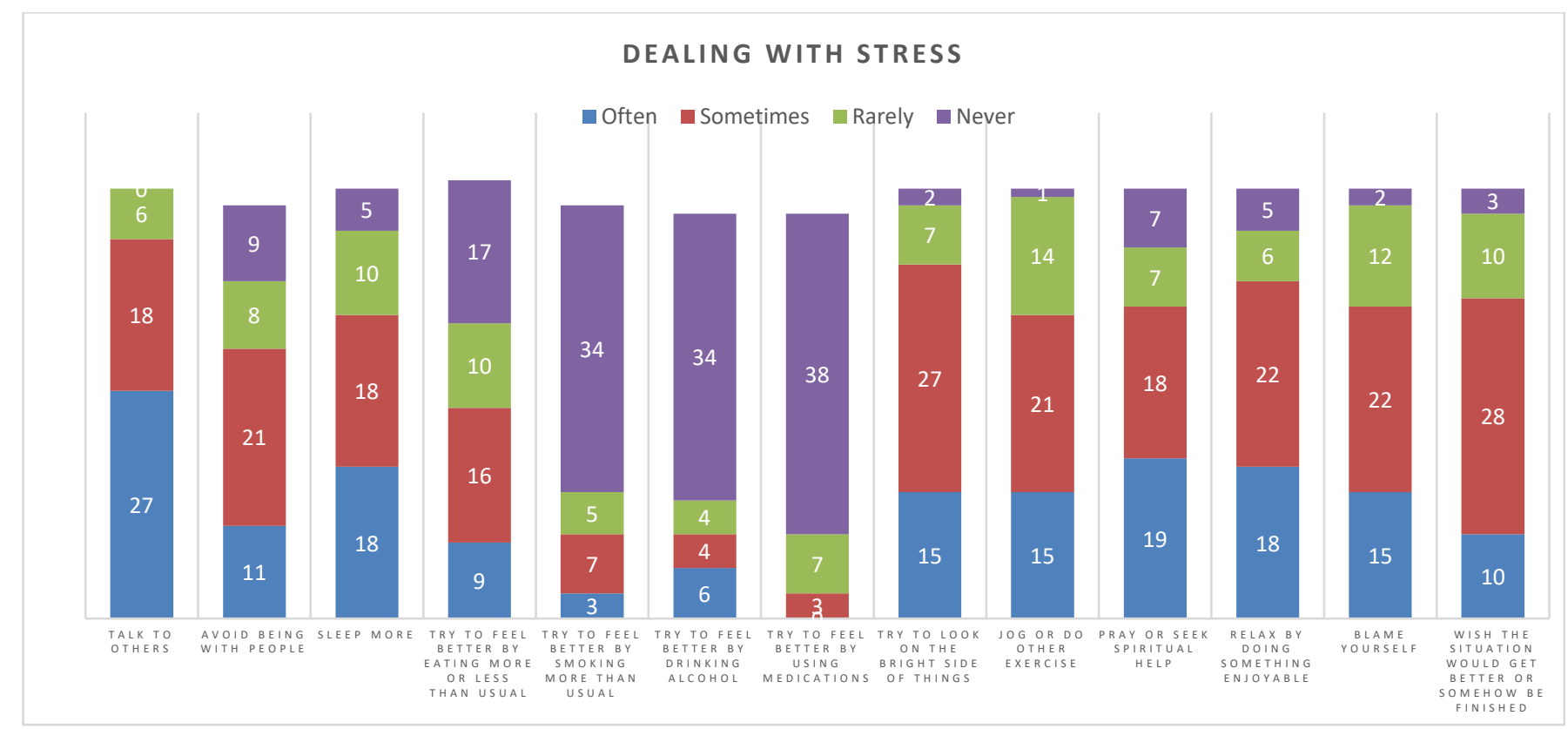

Fig. 2. Participants' coping mechanisms during the last 6 months.

Seventy-four percent $(n=20)$ said that their mental health problems were with no importance", $11 \%(n=3)$ said that their health problems made them seek a psychologist/psychiatrist intervention during the pandemic while none of them had been admitted to a hospital.

The participants had to answer the question "Lately, have you ever felt discouraged to go through with your residency?", $59 \%$ of the respondents $(n=30)$ chose "Yes".

The impact of stress on the wellbeing and the mental health of medical residents and dental residents have been well documented in many dental faculties around the world [3], [5].

Dentistry is known as a stressful profession and dental residents face similar stressors combined with academic pressures that tend to get worse over time.

With this regard, dealing with difficult patients, teaching younger residents, learning complicated clinical and surgical procedures can lead to an important amount of stress on a daily basis.

In addition to those usual stressors, the Covid-19 outbreak has added new challenges to dental residents all over the world.

Tunisia has been severely impacted by the pandemic; school closure, social distancing as well as implementation of safety practices have impacted the training programs at the dental faculty of Monastir, leading to a severe disruption of both the clinical and educational skills procurement.

In the other hand, dental practice exposes practitioners to saliva, blood, and other body fluids, thus the dental residents face an increased risk for contracting the coronavirus [6].

Additional efforts to protect oneself and family members from contamination are to be made by the dental residents on an everyday basis [7] this explains why caring about safety and own health was ranked first when it came to ranking the perceived stressors of the Tunisian dental residents.

Having to deal with lack of personal protection equipment (PPE) was also perceived as stressful. In fact, huge discrepancies in PPE provision to health care workers especially among the public healthcare sectors in middle and low income countries, were reported [8].
Besides, rationing, self-purchasing, substituting, asking patients to purchase PPE, or even having to work without PPE are reported strategies in dealing with the lack of PPE during the pandemic. these strategies can be very stressful and frustrating for young dental residents and can have extremely negative consequences for both workers and patients [9].

A significant correlation between gender and daily stress was found. This can be explained by the fact that Female residents experience all the stressors that their male colleagues face, but they also have to deal with additional stressors unique to them such as balancing the demands of work and home efficiently and being vulnerable to high rates of verbal abuse and physical assault by male patients as well as by other health care workers [10]. One must also wonder whether the results are due to a reporting bias, in that females tend to be more open about their stress than their male counterparts.

The majority of the Tunisian dental residents who took part of this study $(37=74 \%)$ rated their mental health as "good" or "fair". Only four reported "poor" mental health.

In fact, previous studies have described the effects of isolation on mental health in health care workers in general and reported severe stress reactions including physical, cognitive, social, and emotional reactions.

Lee et al. [11] have reported irritability, low mood, insomnia, and posttraumatic stress symptoms among residents and hospital staff after quarantine. These phenomena have a direct negative impact on anxiety and stress. other burn out symptoms such as fatigue, mood disorders, depression and poor patient quality care were reported as well [12].

In our study, the residents confirmed feeling sad and discouraged during le last months. Besides, more than half of the participants revealed feeling discouraged to go through the residency.

Bai et al reported that during the SARS epidemic, being in contact with infected patients was a predictor of developing anxiety, exhaustion, poor concentration, detachment from others, and deteriorating work performance [13]. 
In dentists, the appearance of psychological symptoms has been related to the fear of contracting COVID-19, especially when having a background disease or a higher subjective overload [14]. In fact, based on the nature of the dental procedures, and the proximity of the dental team with patients, it's proven that, in the absence of appropriate protective infection control measurements

The disease could readily spread from infected patients to the dental professional and subsequently to other patients [15].

Surprisingly, although twenty-three (45\%) residents reported having mental health problems during the last months, only 3 of them have consulted a psychologist/psychiatrist. This brings us to the ultimate reluctance and unrecognition of mental health/illness in Tunisia that leads most of those affected with any form of mental disease to avoid consulting. Conveniently, a study Comparing the cultural variations in public beliefs about mental disorders between Tunisia and Germany showed that Tunisians tended much more than Germans to hold moralistic views and to blame the afflicted person for his/her illness" [12].

As a matter of fact, our perception of stress differs from one to another as well as our coping tactics. A moderate amount of pressure can help us perform better in challenging situations, but too much or prolonged stress can lead to physical and psychological distress

With this regards a study held in Saudi Arabia reported that maladaptive stress-coping strategies were associated with high levels of stress among medical residents [16]. Another study of the American pediatric dental residents concluded that residents using positive coping strategies and exercising had lower stress than those using negative ones [17].
In our study, we tried to learn about the surveyed residents' different coping mechanisms. Overall, most of the coping mechanisms chosen were positive. Talking to family and friends, even virtually through social medias, was ranked first, while using medication to feel better was ranked last.

The Table II shows that residents, whether medical or dental, from different parts of the world (Tunisia, Canada, Nigeria) used almost the same coping mechanisms with a slight difference in the spiritual aspect. This could be explained by the fact that spirituality and prayers are more influential in more conservative societies. Taking care of oneself, keeping in touch with family and friends, and finding sources of inspiration and joy are strongly recommended. Social media can assist in communication with relatives, allowing isolated people to receive updates about the situation of loved ones.

\section{LIMITATIONS OF THIS STUDY}

This study has several limitations, especially the small sample size and the low return rate.

Not to forget that some of the answers may be biased since some residents declared being afraid of being identified.

The unavailability of similar studies in Tunisia, also limited the possibility of comparison between different studies with regards to stress levels among the Tunisian dental residents.

Our study could be the base ground and the starting point to future studies tackling the issue of stress among residents from different angles and through different scientific approaches such as the Maslach Burnout Inventory (MBI), or Perceived Stress Scale (PSc).

TABLE II: RANKING OF COPING MECHANISMS: A COMPARISON BETWEEN THE CANADIAN[4], THE NigERIAN[18] STUdiES, AND OUR STUDY

\begin{tabular}{|c|c|c|c|c|}
\hline Coping mechanisms & $\mathrm{N}=$ & $\begin{array}{l}\text { Ranking for } \\
\text { Tunisian dental } \\
\text { residents }\end{array}$ & $\begin{array}{c}\text { Ranking for } \\
\text { Canadian Medical } \\
\text { Residents }\end{array}$ & $\begin{array}{c}\text { Ranking for } \\
\text { Nigerian Medical } \\
\text { Residents }\end{array}$ \\
\hline Talking to others (social media) & 45 & 1 & 1 & 2 \\
\hline Try to look on the bright side of things & 42 & 2 & 3 & 1 \\
\hline Relax by doing something enjoyable & 40 & 3 & 2 & 3 \\
\hline Wish the situation would get better or somehow be finished & 38 & 4 & 5 & 5 \\
\hline Pray or seek spiritual help & 37 & 5 & 10 & 4 \\
\hline Blame yourself & 37 & 6 & 7 & 7 \\
\hline Jog or do other exercises & 36 & 7 & 4 & 9 \\
\hline Sleep more & 36 & 8 & 9 & 8 \\
\hline Avoid being with people & 32 & 9 & 6 & 6 \\
\hline Try to feel better by eating more or less than usual & 25 & 10 & 8 & 10 \\
\hline Try to feel better by smoking more than usual & 10 & 11 & 13 & 13 \\
\hline Try to feel better by drinking alcohol & 10 & 12 & 11 & 12 \\
\hline Try to feel better by using medications & 3 & 13 & 12 & 11 \\
\hline
\end{tabular}

\section{REFERENCES}

[1] Addae HM, Wang X. Stress at work: Linear and curvilinear effects of psychological-, job-, and organization-related factors: An exploratory study of trinidad and tobago. Int J Stress Manag, 2006;13(4):476.

[2] Divaris K, Lai CS, Polychronopoulou A, Eliades T, Katsaros C. Stress and burnout among Swiss dental residents. Schweiz Monatsschr Zahnmed, 2012;122(7-8):610-5. PMID: 22914975.

[3] Jiménez-Ortiz JL, Islas-Valle RM, Jiménez-Ortiz JD, Pérez-Lizárraga E, Hernández-García ME, González-Salazar F Emotional exhaustion, burnout, and perceived stress in dental students. J Int Med Res, 2019 Sep;47(9):4251-4259. doi: 10.1177/0300060519859145. Epub 2019 Jul 16.

[4] Cohen JS. The happy docs study: a Canadian Association of Internes and residents well-being survey examining resident physician health and satisfaction within and outside of residency training in Canada. 2008.

[5] Hung M, Licari FW, Hon ES, Lauren E, Su S, Birmingham WC, Wadsworth LL, Lassetter JH, Graff TC, Harman W, Carroll WB, Lipsky MS. In an era of uncertainty: Impact of COVID-19 on dental education. $J$ Dent Educ. 2021 Feb;85(2):148-156. doi: 10.1002/jdd.12404. Epub 2020 Sep 13. PMID: 32920890.

[6] Zarzecka J, Zarzecka-Francica E, Gala A, Gębczyński K, Pihut M. Dental environmental stress during the COVID 19 pandemic at the Jagiellonian University Medical College, Kraków, Poland. International Journal of Occupational Medicine and Environmental Health, 2021;34(2):211-222. doi:10.13075/ijomeh.1896.01773.

[7] Delgado D, Wyss Quintana F, Perez G, Sosa Liprandi A, PonteNegretti C, Mendoza I, Baranchuk A Personal Safety during the COVID-19 Pandemic: Realities and Perspectives of Healthcare 
Workers in Latin America. Int J Environ Res Public Health, 2020 Apr 18;17(8):2798. doi: 10.3390/ijerph17082798.PMID: 32325718.

[8] Sarfraz A, Sarfraz Z, Anwer A, Sarfraz M, Siddiq J. Availability, Use, and Satisfaction of Personal Protective Equipment Among Healthcare Workers: A Cross-Sectional Assessment of Low- and Middle-Income Countries. J Occup Environ Med, 2020 Nov;62(11):e657-e664. doi: 10.1097/JOM.0000000000002028. PMID: 32956236.).

[9] Ridge LJ, Stimpfel AW, Dickson VV, Klar RT, Squires AP. How clinicians manage routinely low supplies of personal protective equipment. Am J Infect Control, 2021 Aug 18:S0196-6553(21)005460. doi: 10.1016/j.ajic.2021.08.012. Epub ahead of print. PMID: 34416315.

[10] Gautam M. Women in medicine: stresses and solutions. West J Med, 2001;174(1):37-41.

[11] Lee S, Chan LY, Chau AM, et al. The experience of SARS-related stigma at Amoy Gardens. Soc Sci Med, 2005;61(9):2038-2046. doi: 10.1016/j.socscimed.2005.04.010.

[12] Patel RS, Bachu R, Adikey A, et al. Factors related to physician burnout and its consequences: a review. Behav Sci (Basel), 2018;8(11):98. doi: 10.3390/bs8110098.

[13] Buddeberg-Fischer B, Klaghofer R, Stamm M, Siegrist J, Buddeberg C. Work stress and reduced health in young physicians: prospective evidence from Swiss residents. Int Arch Occup Environ Health, 2008;82(1):31-8.

[14] Bhalla N, Suneja N, Kobryn A, Lew S, Dym H. The Psychological Well-Being of Medical Versus Dental GME Residents During the COVID 19 Pandemic: A Cross-Sectional Study. J Oral Maxillofac Surg, 2021;79(9):1828.e1-1828.e8. doi:10.1016/j.joms.2021.04.016.

[15] Banakar M, Bagheri Lankarani K, Jafarpour D et al. COVID-19 transmission risk and protective protocols in dentistry: a systematic review. BMC Oral Health 20, 275 (2020). https://doi.org/10.1186/s12903-020-01270-9.

[16] Alosaimi FD, Almufleh A, Kazim S, Aladwani B. Stress-coping strategies among medical residents in Saudi Arabia: A cross-sectional national study. Pak $J$ Med Sci, 2015;31(3):504-9. doi: 10.12669/pjms.313.7490. PMID: 26150833; PMCID: PMC4485260.

[17] Vergara-Buenaventura A, Chavez-Tuñon M, Castro-Ruiz C. The Mental Health Consequences of Coronavirus Disease 2019 Pandemic in Dentistry. Disaster Med Public Health Prep, 2020;14(6):e31-e34. doi:10.1017/dmp.2020.190).

[18] Ogunsemi O. A survey of perceived stress, intimidation, harassment and well-being of resident doctors in a Nigerian teaching hospital. 2010.

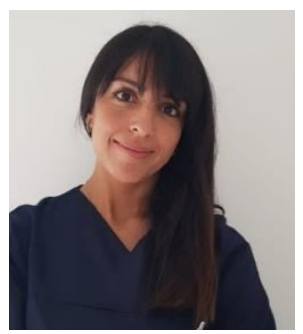

Dr. Dorsaf Touil is doctor of dental medecine, specialist in oral surgery and oral medicine. Currently, she works as a professor assistant in oral medicine and oral surgery, in the department of dentistry at the university Hospital, Sahloul Tunisia. 